\title{
Analysis of Ground Displacement Induced by Double-tube Parallel Shield Tunnels
}

\author{
Weiquan Rong ${ }^{1, a}$, Hong Yuan ${ }^{* 1, b}$, Xiongfei Yang ${ }^{1, c}$ and Jiayu $\mathrm{Wu}^{1, \mathrm{~d}}$ \\ ${ }^{1}$ MOE Key Laboratory of Disaster Forecast and Control in Engineering, Institute of Applied \\ Mechanics, Jinan University, Guangzhou 510632, China. \\ a291037958@qq.com, btyuanhong@jnu.edu.cn, '176048785@qq.com, d1191625174@qq.com
}

Keywords: Double-tube parallel shield tunnels; Ground surface settlement; Soil loss; Numerical analysis

Abstract. Based on Mindlin displacement solution, we fully analyze the mechanical behavior of ground surface settlement caused by tunnel construction. In this paper, modified formula of ground deformation induced by ground loss is offered and applied to estimate settlement induced by the double-line tunnel construction with superposition principle. The successive construction process of double-tube parallel shield tunnels in the same direction was simulated with FLAC3D and the simulated results were compared with values of theoretical formula calculation.

\section{Introduction}

With the large scale development and utilization of underground space, it's common that tunnel construction sites are located in city building dense area, therefore, accurate evaluation of soil displacement caused by tunnel construction is important to building a better security protection scheme for surrounding buildings. At present, there are lots of studies on ground surface settlement. For the single-track tunnel, there are several prediction methods to evaluate the construction settlement as follow: Peck experience formula method, stochastic medium theory, laboratory model tests and Numerical Methods. For tunnel construction, based on Mindlin [1] displacement solution, a method had to be applied for ground surface settlement calculation, taking into account of the excess earth pressure, the lateral friction between shield skin and the surrounding soil and ground loss [2-4]. In this paper, we offer a modified formula for the evaluation of the ground deformation induced by ground loss. Based on Mindlin displacement solution, an improved method for calculation of ground surface settlement is proposed. Eventually, we fully analyze the soil mechanical behavior by using FLAC3D to simulate the construction process of double-line tunnel construction.

2. Calculation of ground surface settlement induced by double-tube parallel shield tunnels construction

Mindlin displacement solution after coordinate conversion. A vertical and horizontal point load are applied at a semi-infinite solid, respectively, as shown in Fig. 1. Through the theory formula deduction, Mindlin (1936) found the displacement in symmetrical cylindrical coordinates. Any point $(x, y, z)$ corresponds to the axis displacements $(u, v, w)$.


Fig. 1 Force normal and parallel to the boundary in the interior of a semi-infinite solid 
Basic assumptions. In order to use classical mathematics and mechanics to calculate the settlement caused by shield construction, we make the following assumptions to simplify the conditions.

(1) In this paper, the TBM (tunnel boring machine) position changes while driving and the "kill" or "alive" of the soils are taking into account. The consolidation of soils is not in consideration;

(2) The model of soils is homogeneous linear elastic semi-infinite solid and the excavation of tunnel construction can't change the boundary of the model;

(3) Shield tunneling machine continues to move uniformly in a straight line, the change of the driving route caused by the correction or rotation of the machine and the angles of tunnel are not in consideration;

(4) During construction, tunnels can't interact with others.

The settlement caused by construction factors. During the construction of double-tube parallel shield tunnels, the influencing factors on ground surface settlement are the excess earth pressure, the lateral friction between shield skin and the surrounding soil, the friction force between cutter-head and soil , the grouting pressure at shied tail and the gravity of the shield machine. Their calculation diagram is shown in Fig. 2.

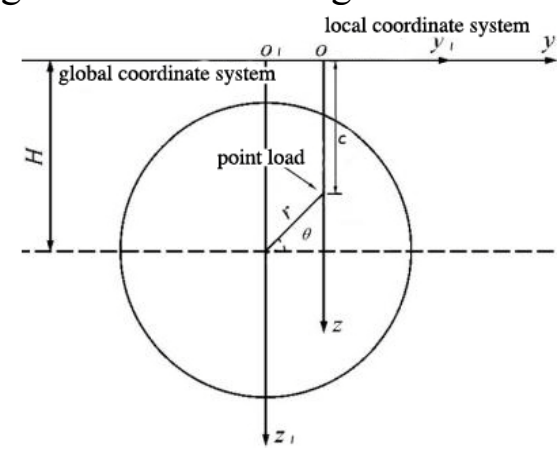

(a)

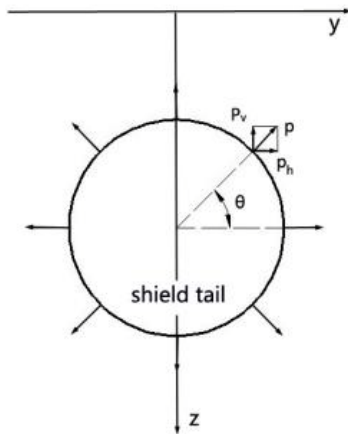

(d)

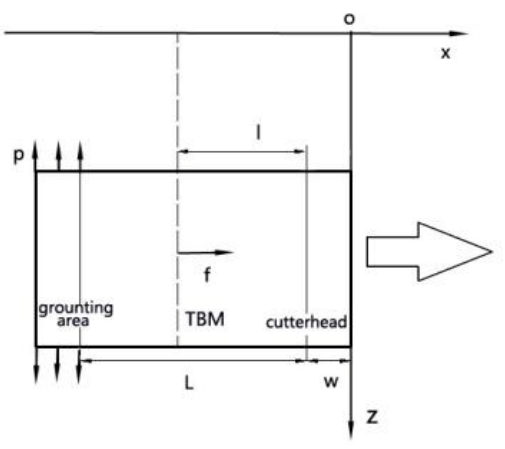

(b)



(e)

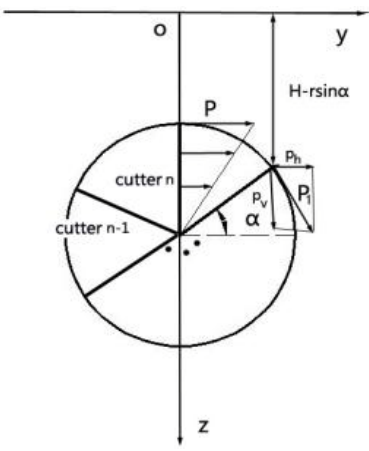

(c)

Fig. 2 Calculation diagram of construction factors. (a) The excess earth pressure; (b) The lateral friction between shield skin and the surrounding soil; (c) The friction force between cutter-head and soil; (d) The grouting pressure at shied tail; (e) The gravity of the shield machine.

The ground settlement caused by strata loss. There are several factors influencing the subsidence and the main driving factor is the deformation caused by strata loss. Combining the research of N. Loganathan et al. [5-6], WEI Gang obtained the subsidence expressions of strata loss which represented the change of Poisson's ratio and the non-equivalent, oval and radial movement of strata.

According to the equivalent parametric method proposed by Lee for the estimated value of subsidence caused by strata loss $V_{\text {loss, }}$, the value of strata loss, can be assigned to the constants $\pi, R$ and $\eta . \eta$ is strata loss ratio.

However, the combination of previous research, the values of widths of settlement tank is smaller than the measured values and can't change with the actual nature of the strata. After a lot of research of strata in London, O. Reilly \& new [7] hold that there was a linear relation between 
the width coefficient $i$ of settlement tank and the depth $H$ of tunnel. Based on above, Hanxuan [8] carried the comparison research on the relationship between actual nature of the strata and the width coefficient. Then, he provided a set of suggestive values $K$, varied from area to area, as the width parameter of settlement tank. Combing with the results above, we obtain the semi-theoretical and semi-empirical expression. The relationship between geological conditions and ground settlement is taking into account.

The total ground surface settlement caused by double-tube parallel shield tunnels. In conclusion, combing with solutions of ground settlement $w(x, y)$ caused by different kinds of construction factors.

In conclusion, combing with solutions of ground settlement caused by different kinds of construction factors, we obtain the total ground surface settlement.

In order to obtain the expression of settlement of double-tube parallel shield tunnels, we superimpose the expression of settlement of single-tube and the calculation diagram is shown in Fig. 3.

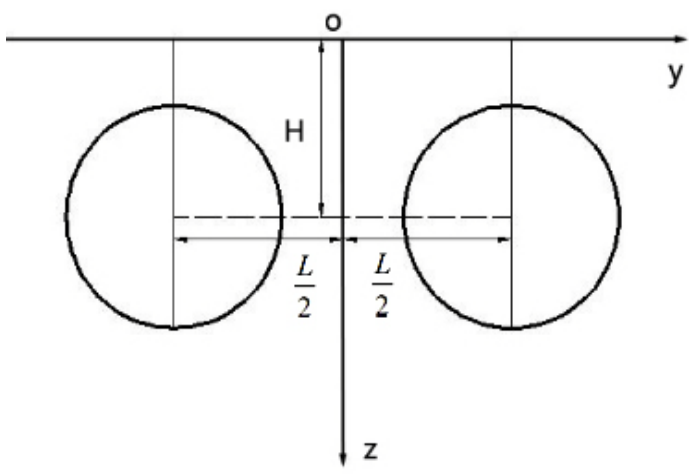

Fig. 3 Calculation diagram of double-tube parallel shield tunnels

The settlement of double-tube parallel shield tunnels excavated at the same time is given by

$$
W(x, y)=w\left(x, y-\frac{L}{2}\right)+w\left(x, y+\frac{L}{2}\right)
$$

\section{Numerical simulation}

Model parameter and grid. Based on the shield construction of the running tunnel of Tianhebei-Wushan project in Guangzhou, the characteristics of transverse and longitudinal surface displacements are studied by numerical simulation with FLAC3D. The outside diameters of segments is $6.0 \mathrm{~m}$ while the inside is $5.4 \mathrm{~m}$. The depth of tunnel in the model is $15 \mathrm{~m}$. The material properties and geometric parameters of model are shown in Table 1.

Table 1 Model material parameter

\begin{tabular}{ccccccc}
\hline layer & $\begin{array}{c}\text { Thickness } \\
(\mathrm{m})\end{array}$ & $\begin{array}{c}\text { Density } \\
(\mathrm{kN} / \mathrm{m} 3)\end{array}$ & $\begin{array}{c}\text { Elastic } \\
\text { modulus } \\
(\mathrm{kPa})\end{array}$ & $\begin{array}{c}\text { Poisson's } \\
\text { ratio }\end{array}$ & $\begin{array}{c}\text { Cohesion } \\
(\mathrm{kPa})\end{array}$ & $\begin{array}{c}\text { Internal } \\
\text { friction } \\
\text { angle }\left({ }^{\circ}\right)\end{array}$ \\
\hline $\begin{array}{c}\text { Miscellaneous fill } \\
\text { Alluvial and diluvial } \\
\text { deposit }\end{array}$ & 1 & 19 & 8200 & 0.43 & 22 & 12 \\
$\begin{array}{c}\text { Hard plastic residual soil } \\
\text { Completely-weathered }\end{array}$ & 3.5 & 19 & 18000 & 0.40 & 23 & 22 \\
$\begin{array}{c}\text { granite } \\
\text { Highly-weathered granite }\end{array}$ & 10 & 19.5 & 23000 & 0.30 & 17 & 21 \\
$\begin{array}{c}\text { Over-break } \\
\text { Shield (steel) }\end{array}$ & 0.07 & 19.8 & 21000 & 0.38 & 28 & 22 \\
Lining segment (C50) & 0.045 & 7850 & 2.06000 & 0.36 & 30 & 25 \\
\hline
\end{tabular}


The parameters of construction are as follows: the excess earth pressure is $-0.3 \mathrm{MPa}$; the ground settlement induced by the friction force between the side of cutter-head and soil is $46 \mathrm{kPa}$; the torque between cutter-head and soil is $2000 \mathrm{kN} \cdot \mathrm{m}$; the grouting pressure at shied tail is $-0.2 \mathrm{MPa}$; the gravity of the shield machine is 520t; the lateral friction between shield skin and the surrounding soil is $8 \mathrm{kPa}$.

The model established in FLAC3D is shown in Fig. 4. Using Mohr-Coulomb elastic-plastic model as constitutive model, the strata and grouting material are created with solid element while the shield and lining segments made out of isotropic material are created with shell element.
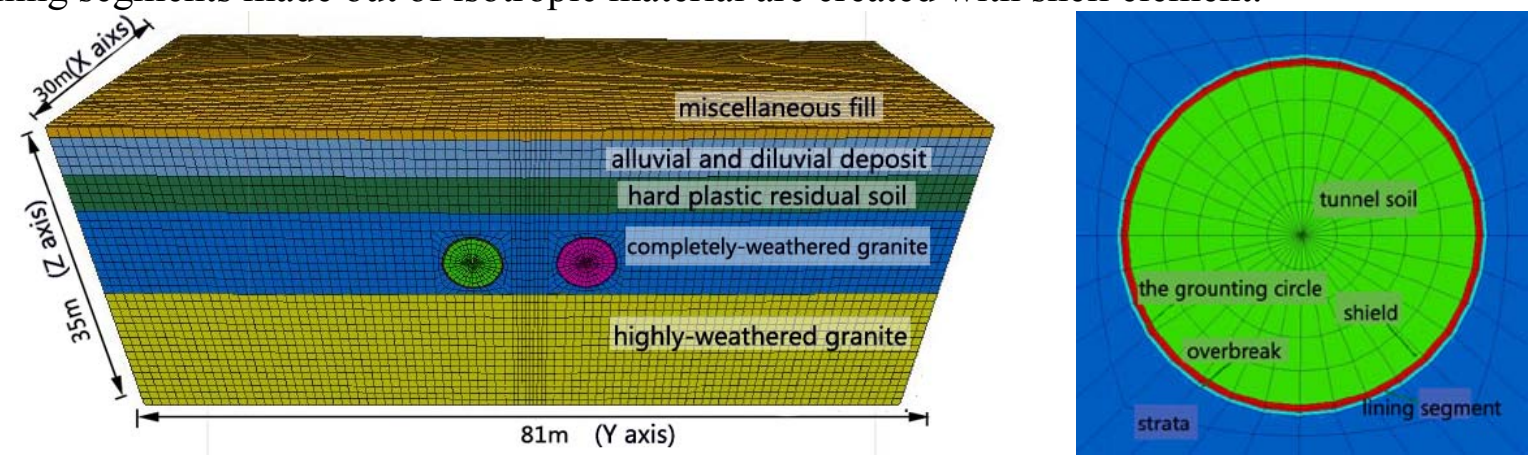

Fig. 4 The classification of soil layer and the grid of surrounding rock of tunnel

Excavation procedure. When modeling, first, the gravity should be applied and its interior stress reach equilibrium under its own weight; second, the initial geos tress field should be reset.

Step 1: Excavate 1.5m along the $\mathrm{Y}$ axis within grouting circle, then applied the excess earth pressure and the gravity of the shield machine. The torque in front of cutter-head is simplified as distributed force produced by 4 lines of cutters. The friction in side of cutter-head is applied surround the layer of overbreak. The distributed force applied surround the shield is provided to simulate part of jacking force for overcoming the friction between shield and soil. Running a certain step. Finally, run a certain time step. The running step determined by trial excavation is the time step that soil on the roof of tunnel need to reach the max manoeuvring space.

Step 2-4: Install shield shells outside the grouting circle and drive 3 rings. There is $7 \mathrm{~cm}$ thickness of disturbed soil surround the shield.

Step 5: Delete the shield shells and the layer of overbraek and apply grouting pressure $0.2 \mathrm{MPa}$ around the strata in $6 \mathrm{~m}-7.5 \mathrm{~m}$ from the tunnel face. Run a certain time step.

Step 6: Delete the grouting pressure and create grouting circle which is as solid as concrete. Run a certain time step.

Step 7: Erect the segments and run a certain time step.

\section{Result comparison between expressions and numerical simulation}

The value of strata loss is determined by calculation as follow. The thickness of shell is $45 \mathrm{~mm}$ and the manoeuvring space of shield tail is $10 \mathrm{~mm}$. According to the study of Lee, strata loss ratio is given by:

The value of strata loss is determined by calculation as follow. The thickness of shell is $45 \mathrm{~mm}$ and the manoeuvring space of shield tail is $10 \mathrm{~mm}$. According to the study of Lee, strata loss ratio $\eta$ is

$$
\eta=\left(4 R g+g^{2}\right) /\left(4 R^{2}\right)=0.92 \%
$$

The value of strata loss $V_{\text {loss }}$ is

$$
V_{\text {loss }}=\alpha G_{p}+w+U_{3 D}=28.9 \mathrm{~mm}
$$

After numerical integration in MATLAB, the solutions introduced above can be seen in Fig. 5 and Fig. 6. 


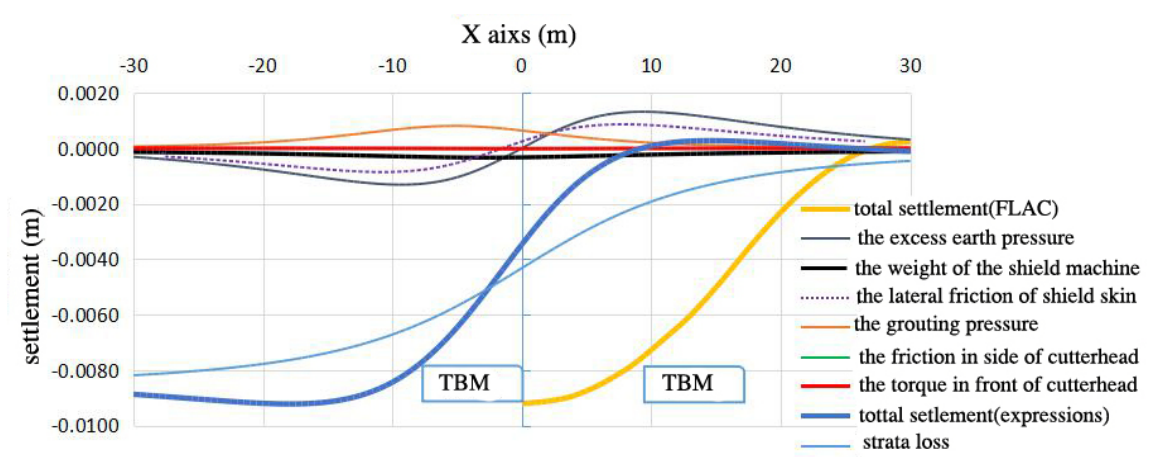

Fig. 5 The result comparison of longitudinal ground settlement between expressions and numerical simulation when the left tunnel advancing $16.5 \mathrm{~m}$



Fig. 6 The result comparison of lateral ground settlement of section $\mathrm{X}=6 \mathrm{~m}$ between expressions and numerical simulation when the left tunnel advancing $22.5 \mathrm{~m}$

The excess earth pressure, the lateral friction between shield skin and the surrounding soil and the grouting pressure have greater impact on ground surface settlement relatively among the factors of tunnel construction, as shown in Fig. 5 and Fig. 6. Longitudinal surface deformation induced by shield tunnel is assumed as an S-shaped curve. Surfaces on both sides hunch up at the axis of excavation face. As shown in analytical calculation, the calculated results of lateral and longitudinal ground settlement is in accordance with the data from numerical simulation, as shown in Fig. 7.

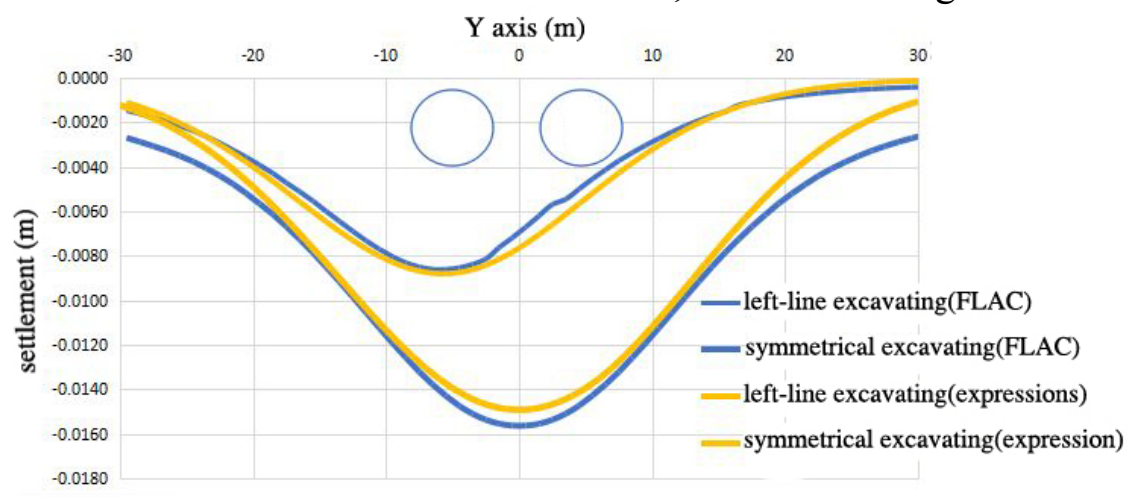

Fig. 7 The result comparison of lateral ground settlement of section $\mathrm{X}=6 \mathrm{~m}$ between expressions and numerical simulation when the double-tube tunnels advancing $22.5 \mathrm{~m}$

Because of ignoring the interaction of two tunnels when calculation, the curves of ground deformation obtained by simple superposition is smaller than those from numerical simulation. The max displacement occur in $\mathrm{Y}=0 \mathrm{~m}$ and the calculated value is $14.9 \mathrm{~m}$ while the numerical simulated is $15.6 \mathrm{~m}$. Two curves are similar and the max error is less than $5 \%$ that is a proof for the reliability of the two methods. 


\section{Conclusion}

Based on the Mindlin displacement solution, this paper fully analyzed the soils mechanical behavior during tunnel construction. In this paper, modified formula of ground deformation induced by ground loss was offered and applied to estimate settlement induced by the double-tube tunnels construction with superposition principle. The successive construction process of double-tube parallel shield tunnels in the same direction was simulated with FLAC3D and we could draw the conclusions as follows:

(1) Based on the Mindlin displacement solution, through the coordinate transforming and mathematical deduction, we can obtain the expression of the ground settlement caused by the factors of tunnel construction respectively. With the modified settlement formula of soil loss and the superposition theory, we can predict the ground surface settlement curve of double-tube parallel shield tunnels .

(2) The process of tunnel construction was simulated with FLAC3D, taking account of the construction parameters, equipment and geological conditions of construction. By comparing the simulated results with analytic solutions, we found that the calculations were consistent with the simulated results.

\section{Acknowledgments}

The authors gratefully acknowledge the financial support provided by the Science and Technology Scheme of Guangzhou City (No. 1563000451), the Science and Technology Scheme of Guangdong Province (No. 2012A030200003), and the National Natural Science Foundation of China (No. $11032005)$.

\section{References}

[1] Mindlin R D. Force at a point in the interior of a semi- infinite solid[J]. Journal of Applied Physics, 1936, 7(5): 195-202.

[2] Wei G, Zhang S, Qi J, et al. Study on calculation method of ground deformation induced by shield tunnel construction[J]. Chinese Journal of Rock Mechanics and Engineering, 2006, 25: 3317-3323.

[3] Wei G, Xu R. Prediction of longitudinal ground deformation due to tunnel construction with shield in soft soil[J]. CHINESE JOURNAL OF GEOTECHNICAL ENGINEERING-CHINESE EDITION-, 2005, 27(9): 1077.

[4] Xiaowu T, Ji Z H U, Wei L I U. Research on soil deformation during shield construction process[J]. Chinese Journal of Rock Mechanics and Engineering, 2010, 29(2): 417-422.

[5] Loganathan N, Poulos H G, Stewart D P. Centrifuge model testing of tunnelling-induced ground and pile deformations[J]. Geotechnique, 2000, 50(3): 283-294.

[6] Loganathan N, Poulos H G. Analytical prediction for tunneling-induced ground movements in clays[J]. Journal of Geotechnical and Geoenvironmental Engineering, 1998, 124(9): 846-856.

[7] O'Reilly M P, New B M. Settlements above tunnels in the United Kingdom - Their magnitude and prediction[M]. 1982.

[8] Han X, Li N, Standing J R. An adaptability study of Gaussian equation applied to predicting ground settlements induced by tunneling in China[J]. Yantu Lixue(Rock and Soil Mechanics), 2007, 28(1): 23-28. 